\title{
Concentrations of phthalate metabolites in children from Poland
}

\section{Kinga Polanska1, Mercè Garí2, Agnieszka Jankowska1, Ewelina Wesolowska1, Wojciech Hanke ${ }^{1}$, Holger M. Koch ${ }^{3}$, Claudia Palmke³, Stephan Bose-O'Reilly²}

${ }^{1}$ Department of Environmental Epidemiology, Nofer Institute of Occupational Medicine (NIOM), Lodz, Poland 2Institute and Clinic for Occupational, Social and Environmental Medicine University Hospital (LMU), Munich, Germany 3Institute of the Ruhr-University Bochum (IPA), Bochum, Germany

Phthalates are frequently used as plasticizers and solvents and thus are widely present in our environment Low-molecular weight phthalates (Low-MWP $<250 \mathrm{~g} / \mathrm{mol}$ )

High-molecular weight phthalates (High-MWP $>250 \mathrm{~g} / \mathrm{mol}$ )

The aim of this study was to determine urinary levels of phthalate metabolites in children in Poland

Methods

Polish Mother and Child Cohort REPRO_PL
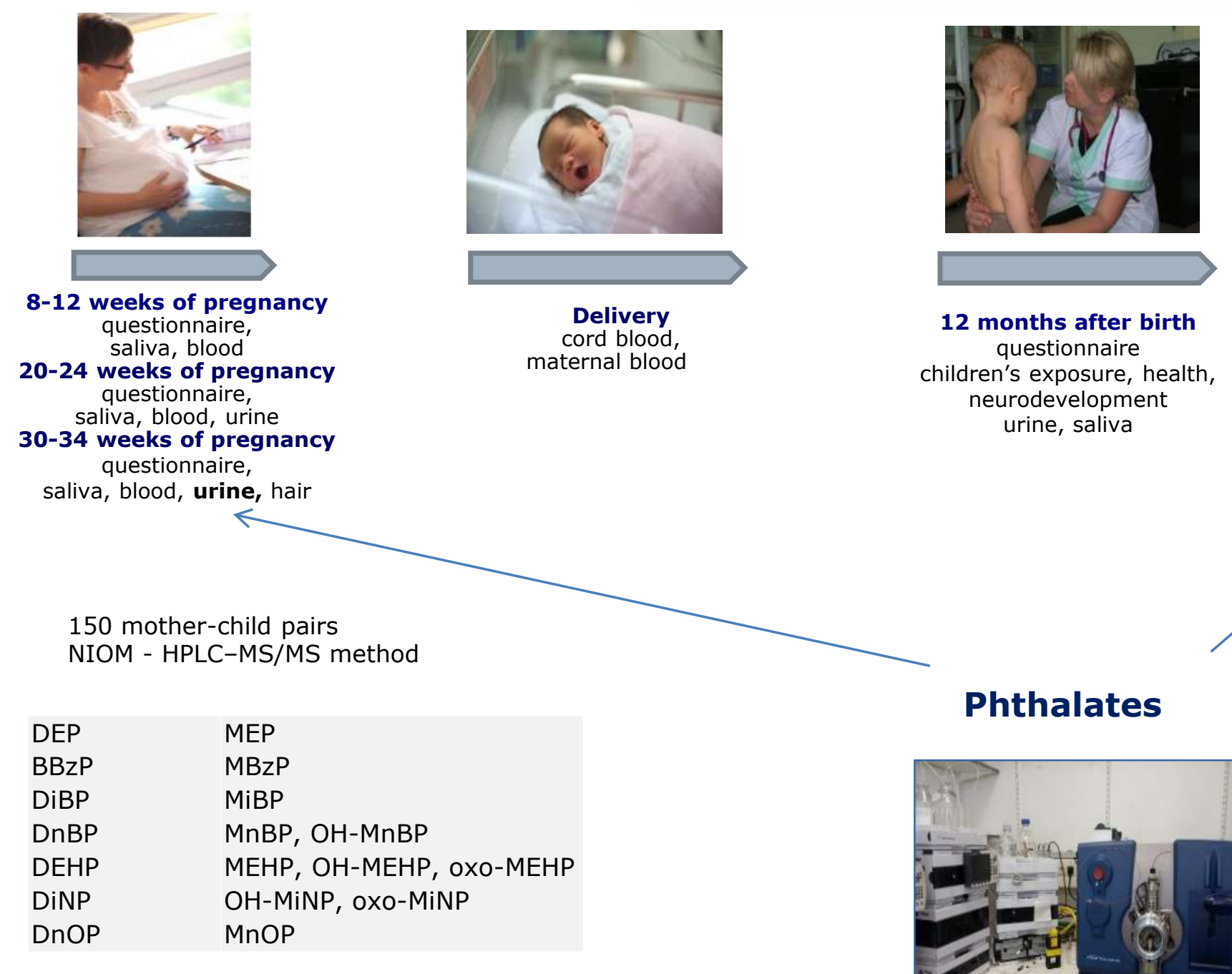

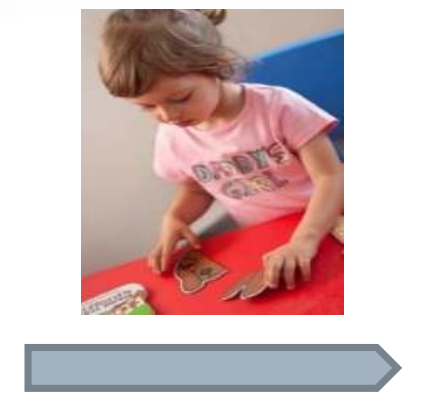

24 months after birth

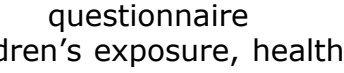
neurodevelopment urine, saliva
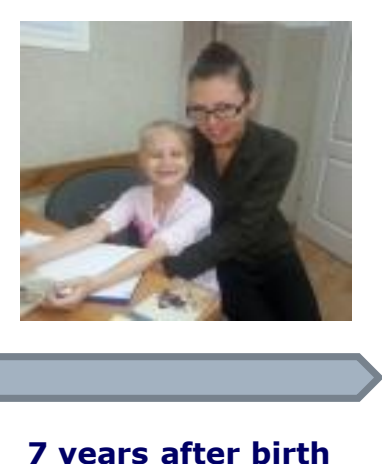

questionnaire, children's exposure, health, urine, saliva, buccal swab
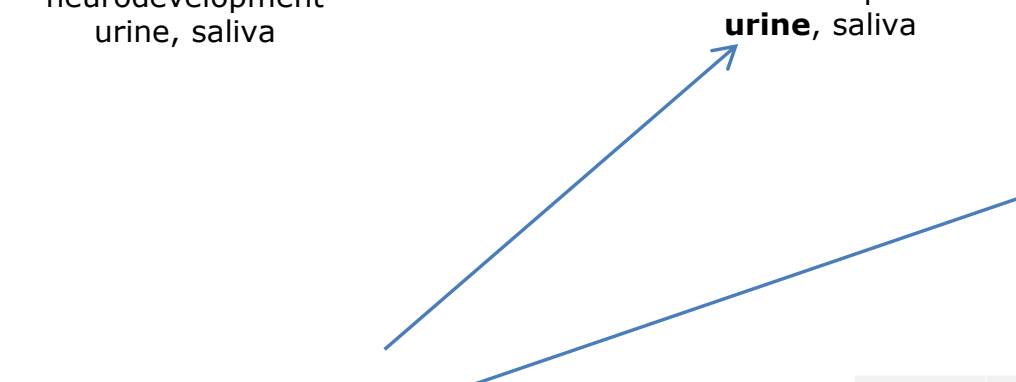

Phthalates

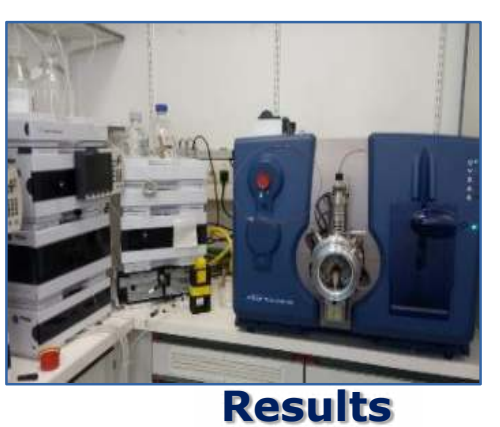

IPA - HPLC-MS/MS method

Concentrations of phthalate metabolites in urine of mothers (3rd trimester) and children ( 2 years of age)

Concentrations of phthalate metabolites in urine of 7 year-old children depending on socio-demographic characteristics

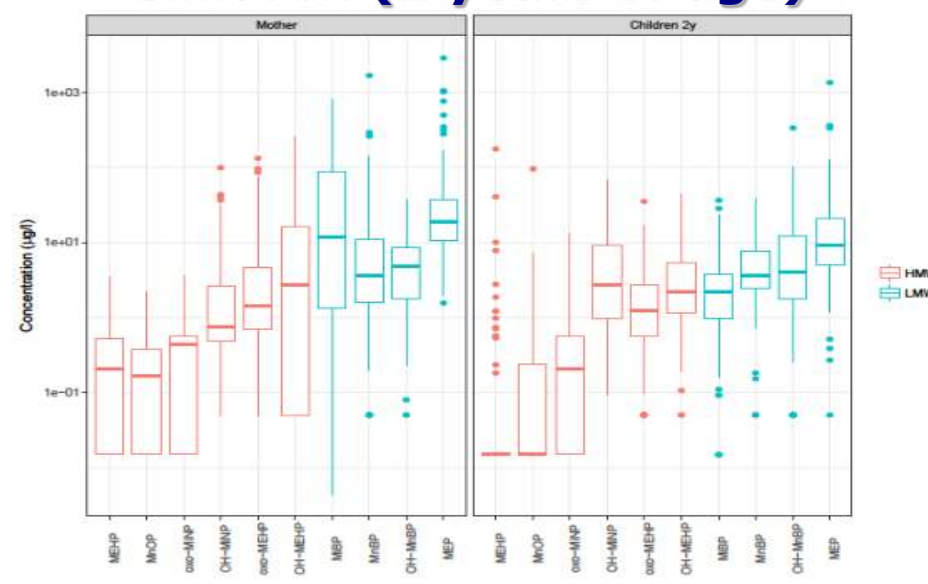

Concentrations of phthalate metabolites in urine of 7 year-old children

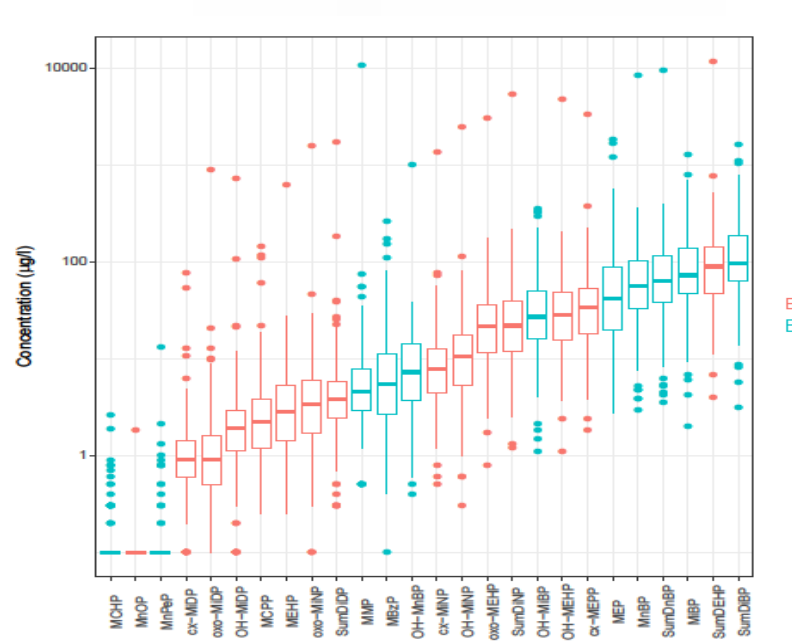

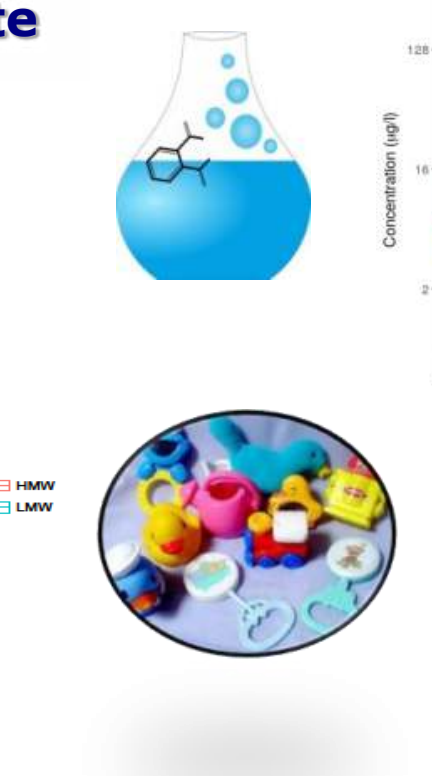

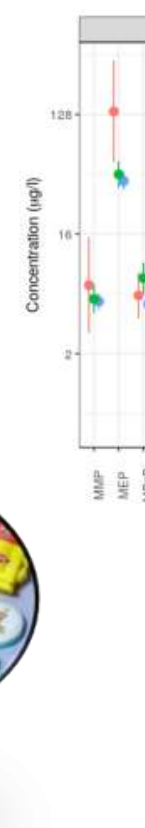

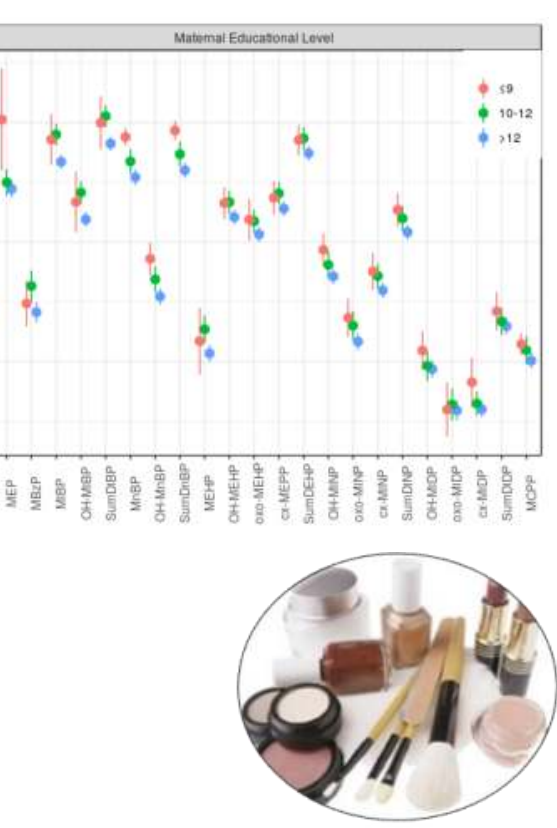

\section{Conclusions}

$\square$ The most abundant compounds belong to the LMW phthalates: MiBP, MnBP and MEP

$\square$ The highest concentrations of the phthalate metabolites were observed among children from the families with lower SES

$\square$ The levels were similar to those found in previous studies performed in Poland

$\square$ The levels of DEHP metabolites in the present study were similar to those observed among children in comparable age from Slovakia, Czech Republic, Romania and Spain but higher than those from other European populations 